\title{
Use of phosphodiesterase type 5 inhibitors and risk of melanoma: a meta-analysis of observational studies
}

This article was published in the following Dove Press journal:

OncoTargets and Therapy

\author{
Xinming Han' \\ Yan $\mathrm{Han}^{2}$ \\ Yongsheng Zheng' \\ Qiang Sun' \\ Tao $\mathrm{Ma}^{\prime}$ \\ Li Dai' \\ Junyi Zhang' \\ Lianji $\mathrm{Xu} \mathrm{u}^{\prime}$
}

'Plastic Surgery Department, Beijing Tongren Hospital, Capital Medical University, Beijing, ${ }^{2}$ Plastic and Reconstructive Surgery Department, Chinese PLA General Hospital, Beijing, China
Correspondence: Yongsheng Zheng Plastic Surgery Department, Beijing Tongren Hospital, Capital Medical University, No 8 Chongwenmen Nei Street, Beijing 100005, China $\mathrm{Tel} / \mathrm{fax}+86 \quad 1058269261$ Email yszheng2017@sina.com
Background: Phosphodiesterase type 5 inhibitor (PE5i) administration may stimulate the proliferation and survival of melanocytes. However, discrepancies remain regarding the association between PDE5i use and melanoma risk in observational studies in humans.

Aim: To evaluate the association between PDE5i use and melanoma in a meta-analysis.

Materials and methods: Studies were identified by searching the PubMed and Embase databases. A random-effects model was applied to synthesize the data. A stratified study was performed to evaluate the influence of study characteristics on outcomes.

Results: Four prospective cohort studies and three case-control studies with 1,534,615 male participants and 16,053 melanoma cases were incorporated. Patients who received a PDE5i had a significantly increased risk for melanoma (adjusted risk ratio $[\mathrm{RR}]=1.12,95 \% \mathrm{CI}=1.03-1.33$, $P=0.008)$ with moderate heterogeneity $\left(I^{2}=54 \%\right)$. Cohort studies (adjusted RR $=1.22,95 \%$ $\mathrm{CI}=1.02-1.46, P=0.03$ ) largely contributed to this result rather than case-control studies. Subsequent stratified analyses revealed that sildenafil was associated with an increased risk of melanoma (adjusted $\mathrm{RR}=1.26,95 \% \mathrm{CI}=1.07-1.50, P=0.007$ ), but tadalafil and vardenafil were not. Also, PDE5i use was associated with a significantly increased risk of in situ melanoma (adjusted $\mathrm{RR}=1.31,95 \% \mathrm{CI}=1.01-1.69, P=0.04$ ), but not of localized or nonlocalized melanoma.

Conclusion: PDE5i use may be associated with a significantly increased risk for melanoma in men. However, further research is needed to determine whether the association is causative.

Keywords: phosphodiesterase type 5 inhibitors, melanoma, meta-analysis, sildenafil

\section{Introduction}

Melanoma is a malignant tumor that has been correlated with endogenous factors such as skin-eye-hair color, family history, and the presence of common or atypical nevi, as well as exogenous factors such as excessive sun exposure. ${ }^{1}$ Biologically, many dysfunctions of molecular pathways have been demonstrated to be involved in the pathogenesis of melanoma, and the activation of the RAS/RAF/mitogen-activated protein kinase and extracellular signal-regulated kinase (ERK) kinase (MEK)/ERK signaling pathway is one of the main key signals that determine the pathogenesis and progression of melanoma. ${ }^{1-3}$ Moreover, activation of the RAS/RAF/ERK/MEK pathway has been recognized to be a result of BRAF somatic mutation (OMIM*164757), which causes the downregulation of cyclic guanosine monophosphate (cGMP)-specific phosphodiesterase (PDE) 5A and lowers cGMP degradation, leading to an increase in intracellular $\mathrm{Ca}^{2+}{ }^{2-6}$ These mechanisms have been considered to be important for the invasion and metastasis of melanocytes, and treatments targeting these signaling 
pathways have shown promising therapeutic efficacy in patients with melanoma. ${ }^{78}$

Phosphodiesterase type 5 inhibitors (PDE5is), including sildenafil, tadalafil, and vardenafil, are a category of medications approved for the treatment of erectile dysfunction. ${ }^{9-11}$ These medications function as inhibitors of cGMP-specific PDE5A, which mimic the effects of the RAS/RAF/ERK/ MEK pathway and may thereby contribute to the development and progression of melanoma. Indeed, some experimental studies in cell lines in vitro have indicated that the administration of a PDE5i was associated with enhanced proliferation and survival of melanocytes. ${ }^{12-14}$ However, the results of subsequent observational studies regarding the relationship between PDE5i use and melanoma risk were not always consistent. ${ }^{15-20}$ The discrepancies of these results may be due to the differences in the included participants, regimens of PDE5i administration, and limited sample sizes of some related studies, which may be underpowered to detect a significant result. ${ }^{21}$ Therefore, in this study, we performed a meta-analysis of observational studies to elucidate the association between PDE5i use and risk of melanoma. Moreover, we also aimed to evaluate whether the relationship between PDE5i use and risk of melanoma was significantly affected by the individual application of PDE5i.

\section{Materials and methods}

The Meta-analysis of Observational Studies in Epidemiology ${ }^{22}$ and Cochrane's Handbook guidelines ${ }^{23}$ were followed during the performance of this meta-analysis.

\section{Database searching}

The PubMed and Embase databases were systematically searched for potential relevant records using the following searching terms: "phosphodiesterase type 5 inhibitors", "PDE5 inhibitors", "PDE5i”, "avanafil”, "lodenafil”, “mirodenafil”, "sildenafil", "tadalafil", "udenafil", or "vardenafil”, combined with "melanoma". This search was limited to human studies and English language publications. The references of the original and review articles were also manually analyzed. The final literature search was performed on April 14, 2017.

\section{Inclusion and exclusion criteria}

Articles were included in this meta-analysis if the following criteria were met: 1) published as full-length articles or abstracts of scientific sessions in English; 2) reported as cohort studies or case-control studies; 3 ) included an adult population of $\geq 18$ years of age; 4 ) identified use of PDE5i as exposure of interest; 5) included those not treated with a PDE5i as controls; 6) compared the risk of melanoma between participants who used a PDE5i vs those who did not; 7) reported the adjusted hazards ratio, relative risk (RR), or odds ratio and their corresponding 95\% CIs for melanoma risk between the PDE5i users and controls. For studies that included overlapping participants, those with a larger sample size were included in the meta-analysis. Irrelevant studies, editorials, review articles, and experimental studies in cell lines or animals were excluded.

\section{Data extraction and quality evaluation}

Two authors independently performed the literature search, data extraction, and quality assessment according to the predefined inclusion criteria. Discrepancies were resolved by consensus. The extracted data included the following: 1) name of the first author, year of publication, and country where the study was conducted; 2) the general characteristics and source of the study participants; 3 ) the range of the study years; 4) the total number of participants, PDE5i users, and melanoma cases; 5) the follow-up durations for the included cohort studies; 6) the strategies used to confirm a diagnosis for melanoma; and 7) the adjusted variables for reporting the association between PDE5i use and melanoma risk. The quality of each study was evaluated using the NewcastleOttawa Scale, ${ }^{24}$ which ranged from 1 to 9 stars, and each study was evaluated based on three aspects: the selection of the study groups, the comparability of these groups, and the ascertainment of the outcome of interest.

\section{Statistical analyses}

RR was used as the general measure for the association between the use of PDE5i and risk of melanoma across studies, and the most adequately adjusted data were extracted for subsequent meta-analyses. The data of RRs and their corresponding standard errors were calculated from $95 \%$ CIs or $P$-values, and were logarithmically transformed to stabilize variance and normalize the distribution. ${ }^{23}$ Cochrane's $Q$-test and $I^{2}$-test were applied to evaluate the heterogeneity among the included studies. ${ }^{25}$ Significant heterogeneity was confirmed if $I^{2}$ was $>50 \%$. A random-effects model was used to synthesize the RR data, because this model is expected to retrieve a more generalized result via the incorporation of potential heterogeneity. ${ }^{23}$ Sensitivity analyses were performed by removing one study at a time to test the robustness of the results. ${ }^{26}$ Stratified analyses were performed to evaluate whether the associations between PDE5i use and melanoma risk were different according to different study characteristics such as the study design (cohort studies or case-control studies), the timing of PDE5i administration (recent use or early use), the number of prescriptions (the lowest or 
highest prescription categories), the individual PDE5i used (sildenafil, tadalafil, or vardenafil), and the stages of melanoma evaluated (in situ, localized, or nonlocalized). Since the definitions of the recent use of PDE5i were not consistent among the included studies, the definitions included from the original articles were used. In addition, since the cut-offs for the categorization of the PDE5i prescriptions were also not consistent among these included studies, the definitions from the original articles were used, and the associations between PDE5i use and melanoma risk were summarized in the lowest and highest prescription categories, respectively. Potential publication bias was assessed by funnel plots using Egger regression asymmetry test. ${ }^{27}$ RevMan (Version 5.1; Cochrane Collaboration, Oxford, UK) and STATA software (Version 12.0; Stata Corporation, College Station, TX, USA) were used for the meta-analysis and statistical analysis.

\section{Results}

\section{Literature search}

The literature search process is presented in Figure 1. Briefly, 726 articles were identified via the initial literature search in the PubMed and Embase databases, and 706 articles were excluded after the screening of titles and abstracts, mainly due

Records identified through database searching $(n=126)$

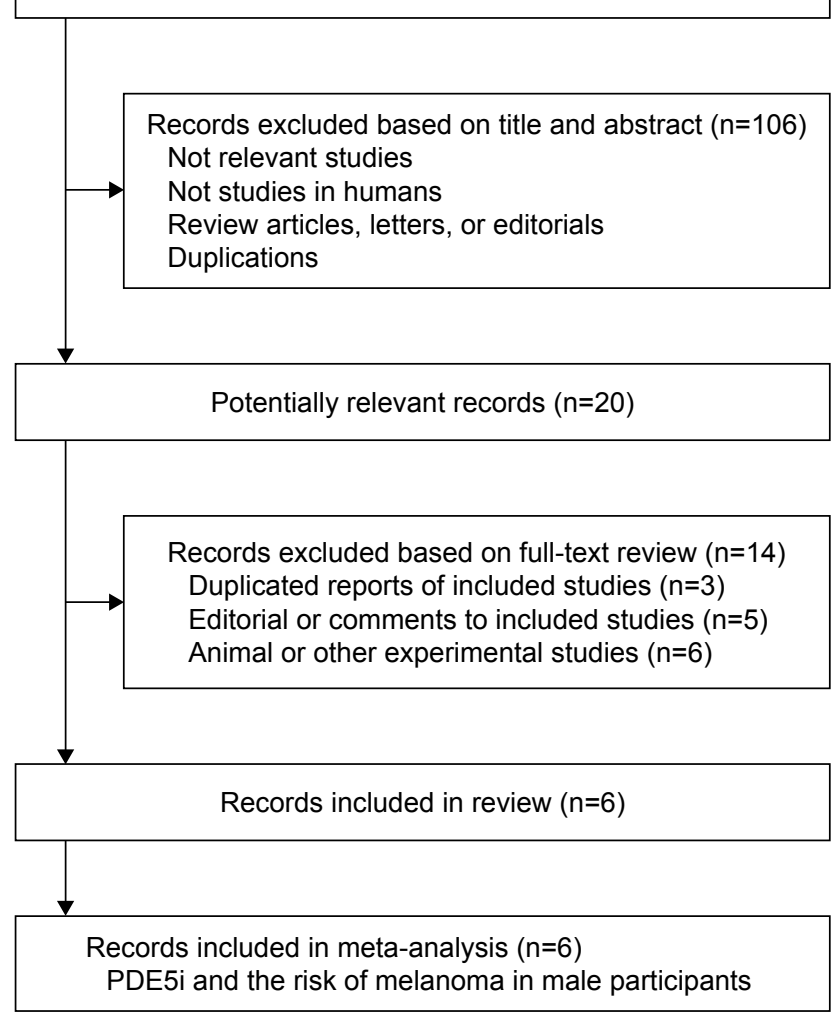

Figure I Flowchart of study selection.

Abbreviation: PDE5i, phosphodiesterase type 5 inhibitor. to the irrelevance of these articles to the purpose of this study. Subsequently, 20 potential relevant articles underwent fulltext review. Among these, 14 articles were further excluded. Among these excluded articles, three articles were duplicate reports, five articles were editorial or comments, and six articles were experimental studies. Finally, six articles ${ }^{15-20}$ were included in the meta-analysis.

\section{Characteristics and quality of the included studies}

Since one of the included articles ${ }^{20}$ reported the data of two case-control studies (the Danish Nationwide Health Registries and the Kaiser Permanente Northern California), a total of seven studies with 1,534,615 participants and 16,053 melanoma cases were incorporated into our metaanalysis. ${ }^{15-20}$ The characteristics and quality scores of these included studies are summarized in Table 1. All of these studies were published after 2014. Among these, four studies were designed as cohort studies, ${ }^{15,17-19}$ one study was a nested case-control study, ${ }^{16}$ and two studies were case-control studies. ${ }^{17}$ Furthermore, among these included studies, three studies were conducted in the USA, ${ }^{15,17,20}$ two studies in the UK, ${ }^{18,19}$ and two studies in Northern Europe. ${ }^{16,20}$ As for the general characteristics of the included participants, six studies included males from the general population ${ }^{15-17,19,20}$ and one study included males with erectile dysfunction. ${ }^{18}$ The mean age of the included participants ranged from 57.0 to 74.5 years, and the indexed study year ranged from 1998 to 2015. Regarding the investigation of individual PDE5i, sildenafil users were analyzed in all of the included studies, while tadalafil and vardenafil users were evaluated in five studies. Melanoma diagnosis was confirmed according to the International Classification of Diseases version 9 or 10, or with the information of national cancer registries. All studies were adjusted for multiple variables (at least for age and race) in reporting the association between the use of PDE5i and melanoma risk. The Newcastle-Ottawa scale scores ranged from 6 to 9 in the included cohort studies.

\section{Overall results for the association between PDE5i use and melanoma risk}

The pooled results of the six studies were analyzed with the random-effects model to evaluate the overall association between PDE5i use and risk of melanoma. The results revealed that PDE5i treatment was associated with a significantly increased risk for melanoma (adjusted $\mathrm{RR}=1.12$, 95\% CI $=1.03-1.33, P=0.008$; Figure 2A). Furthermore, considerable heterogeneity was detected among the included studies ( $P$ for Cochrane's $Q$-test $=0.05, I^{2}=54 \%$ ). Sensitivity 


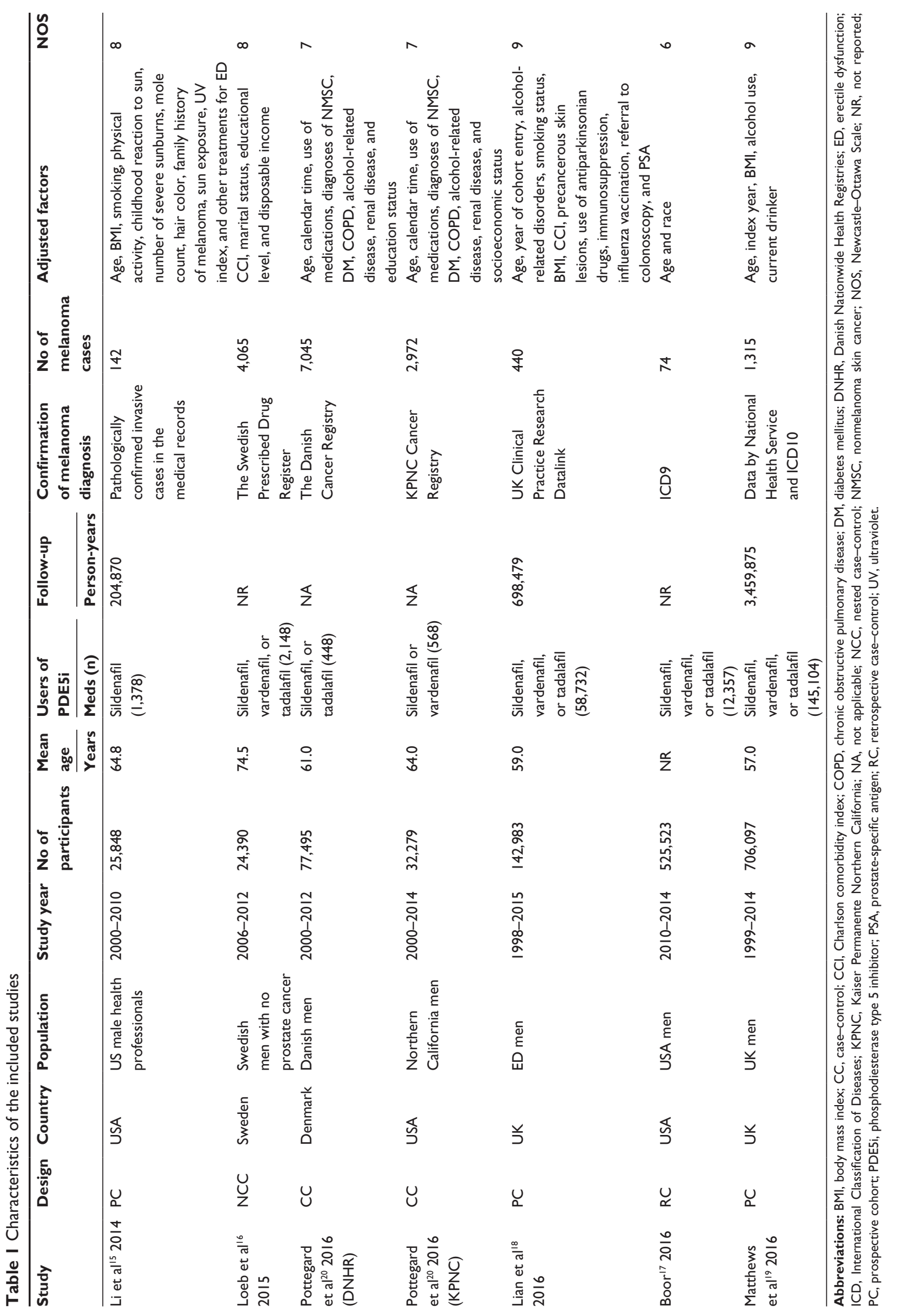




\begin{tabular}{|c|c|c|c|}
\hline $\begin{array}{l}\text { Study or } \\
\text { subgroup }\end{array}$ & $\log (R R)$ & SE & $\begin{array}{l}\text { Weight } \\
(\%)\end{array}$ \\
\hline Li et al ${ }^{15}$ & 0.6523 & 0.2649 & 2.5 \\
\hline Loeb et $a^{16}$ & 0.1906 & 0.0588 & 21.1 \\
\hline Pottegard et $\mathrm{al}^{20}$ (DNHR) & 0.0583 & 0.0526 & 22.9 \\
\hline Pottegard et $\mathrm{al}^{20}$ (KPNC) & 0.01 & 0.053 & 22.8 \\
\hline Lian et $a^{18}$ & 0.1655 & 0.1114 & 10.6 \\
\hline Matthews et al ${ }^{19}$ & 0.131 & 0.0624 & 20.1 \\
\hline \multicolumn{3}{|l|}{ Total $(95 \% \mathrm{Cl})$} & 100 \\
\hline \multicolumn{4}{|c|}{$\begin{array}{l}\text { Heterogeneity: } \tau^{2}=0.01 ; \chi^{2}=10.83, d f=5(P=0.05) ; I^{2}=54 \% \\
\text { Test for overall effect: } Z=2.67(P=0.008)\end{array}$} \\
\hline
\end{tabular}

RR RR IV,

IV, random, $95 \% \mathrm{Cl} \quad$ random, $\mathbf{9 5 \%} \mathrm{CI}$

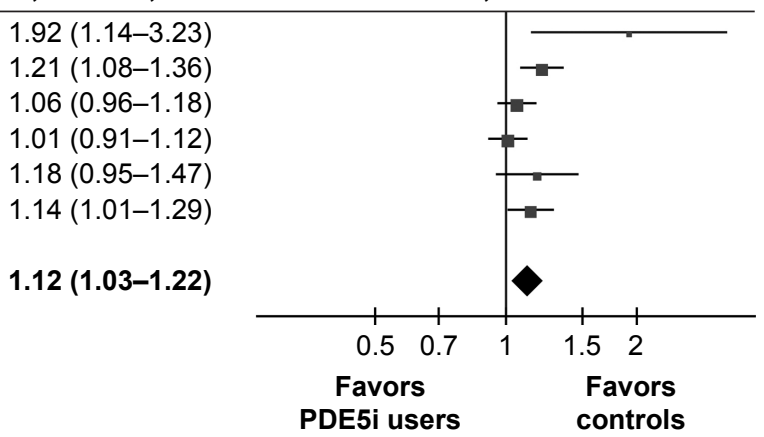

\begin{tabular}{|c|c|c|c|}
\hline $\begin{array}{l}\text { Study or } \\
\text { subgroup }\end{array}$ & $\log (R R)$ & SE & $\begin{array}{l}\text { Weight } \\
(\%)\end{array}$ \\
\hline \multicolumn{4}{|l|}{ Cohort studies } \\
\hline Li et al ${ }^{15}$ & 0.6523 & 0.2649 & 2.5 \\
\hline Lian et $\mathrm{al}^{18}$ & 0.1655 & 0.1114 & 10.6 \\
\hline Matthews et $\mathrm{al}^{19}$ & 0.131 & 0.0624 & 20.1 \\
\hline \multicolumn{4}{|c|}{$\begin{array}{l}\text { Subtotal }(95 \% \mathrm{Cl}) \\
\text { Heterogeneity: } \tau^{2}=0.01 ; \chi^{2}=3.67, d f=2(P=0.16) ; I^{2}=46 \%\end{array}$} \\
\hline \multicolumn{4}{|c|}{ Test for overall effect: $Z=2.15(P=0.03)$} \\
\hline \multicolumn{4}{|l|}{ Case-control studies } \\
\hline Loeb et al $^{16}$ & 0.1906 & 0.0588 & 21.1 \\
\hline Pottegard et $\mathrm{al}^{20}$ (DNHR) & 0.0583 & 0.0526 & 22.9 \\
\hline Pottegard et $\mathrm{al}^{20}$ (KPNC) & 0.01 & 0.053 & 22.8 \\
\hline \multicolumn{4}{|c|}{ Heterogeneity: $\tau^{2}=0.01 ; \chi^{2}=5.45, d f=2(P=0.07) ; I^{2}=63 \%$} \\
\hline \multicolumn{4}{|c|}{ Test for overall effect: $Z=1.60(P=0.11)$} \\
\hline \multicolumn{4}{|c|}{$\begin{array}{l}\text { Total }(95 \% \text { Cl) } \\
\text { Heterogeneity: } \tau^{2}=0.01 ; \chi^{2}=10.83, d f=5(P=0.05) ; l^{2}=54 \%\end{array}$} \\
\hline $\begin{array}{l}\text { Test for overall effect: } Z=2 \\
\text { Test for subgroup differen }\end{array}$ & $\begin{array}{l}7(P=0.008) \\
\mathrm{s}: \chi^{2}=1.15, c\end{array}$ & 列 & - \\
\hline
\end{tabular}

RR RR IV, IV, random, $95 \% \mathrm{Cl} \quad$ random, $95 \% \mathrm{CI}$

$1.92(1.14-3.23)$

$1.18(0.95-1.47)$

$1.14(1.01-1.29)$

1.22 (1.02-1.46)
$1.21(1.08-1.36)$

$1.06(0.96-1.18)$

$1.01(0.91-1.12)$

$1.09(0.98-1.20)$
$1.12(1.03-1.22)$

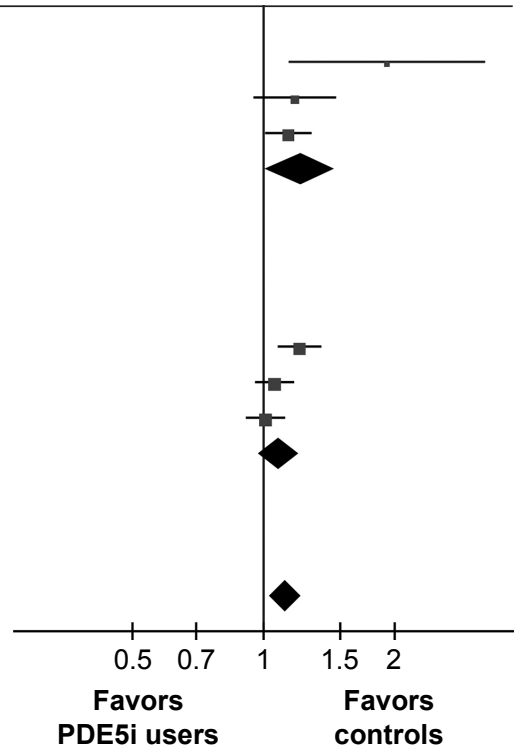

Figure 2 Forest plots for the meta-analysis of the association between PDE5i use and risk of melanoma presented as adjusted RR.

Notes: (A) Forest plots for all available observational studies; (B) forest plots stratified by study design (cohort studies or case-control studies). The effect size of each study is proportional to the statistical weight. The diamond indicates the overall summary estimate for the analysis; the width of the diamond represents the $95 \% \mathrm{Cl}$.

Abbreviations: $d f$, degrees of freedom; DNHR, Danish Nationwide Health Registries; IV, inverse variance; KPNC, Kaiser Permanente Northern California; PDE5i, phosphodiesterase type 5 inhibitor; RR, risk ratio; SE, standard error.

analyses conducted by omitting one study at a time did not significantly change these results, suggesting the robustness of the results. Subsequent subgroup analyses by study design revealed that PDE5i use was associated with a significantly increased risk for melanoma in these cohort studies (three studies, ${ }^{15,18,19}$ adjusted RR $=1.22,95 \% \mathrm{CI}=1.02-1.46$, $P=0.03, I^{2}=46 \%$; Figure $\left.2 \mathrm{~B}\right)$, but the association was not significant in the pooled results for three case-control studies $^{16,20}$ (adjusted RR $=1.12,95 \% \mathrm{CI}=1.03-1.22, P=0.11$, $I^{2}=63 \%$; Figure $2 \mathrm{~B}$ ).

\section{Stratified analysis of the association between PDE5i use and risk of melanoma}

A stratified analysis was performed according to the timing of PDE5i use, the number of prescriptions, the individual
PDE5I used, and the stage of melanoma. Two studies ${ }^{15,16}$ provided stratified data according to the timing of PDE5i use, and these pooled results revealed that recent use of PDE5i was associated with a significantly increased risk for melanoma (adjusted RR $=1.38,95 \% \mathrm{CI}=1.02-1.86$, $P=0.04, I^{2}=35 \%$; Figure $3 \mathrm{~A}$ ), whereas early use of these medications was not (adjusted $\mathrm{RR}=1.46,95 \% \mathrm{CI}=0.76-2.80$, $P=0.26, I^{2}=77 \%$; Figure $3 \mathrm{~A}$ ). Moreover, the stratified analysis of five studies ${ }^{16,18-20}$ indicated that PDE5i use was associated with an increased risk of melanoma in both the lowest (adjusted RR $=1.10,95 \% \mathrm{CI}=1.00-1.20, P=0.04$, $I^{2}=40 \%$; Figure $3 \mathrm{~B}$ ) and highest (adjusted $\mathrm{RR}=1.14,95 \%$ $\mathrm{CI}=1.01-1.29, P=0.04, I^{2}=32 \%$; Figure $3 \mathrm{~B}$ ) prescription categories. Further analyses regarding the individual use of PDE5i revealed that the use of sildenafil was associated with 


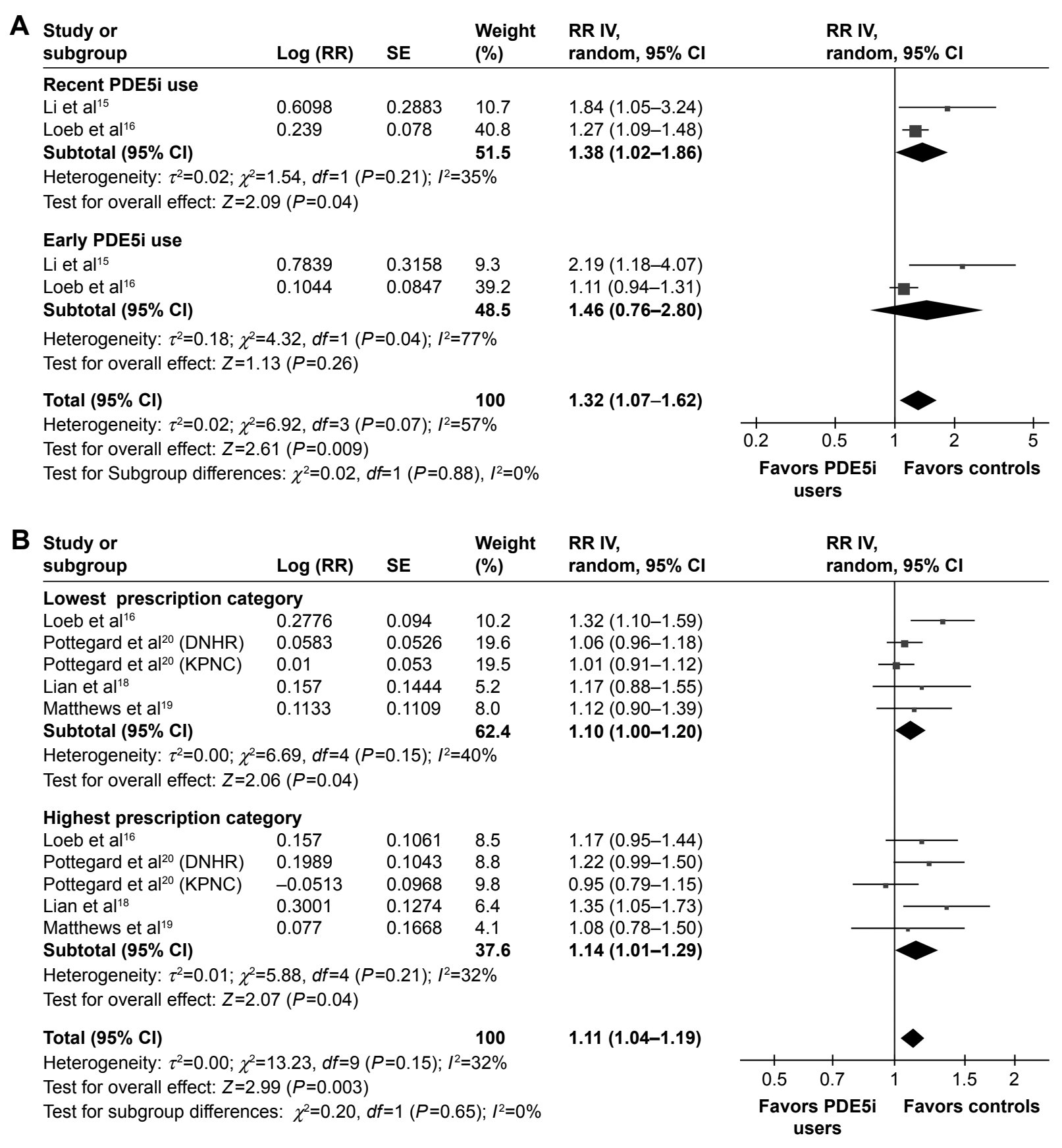

Figure 3 Forest plots for the meta-analysis of the association between PDE5i use and risk of melanoma presented as adjusted RR.

Notes: (A) Forest plots stratified by the timing of PDE5i administration (recent or early use of PDE5i); (B) forest plots stratified by the categories of PDE5i prescription (lowest or highest prescription category). The effect size of each study is proportional to the statistical weight. The diamond indicates the overall summary estimate for the analysis; the width of the diamond represents the $95 \% \mathrm{Cl}$.

Abbreviations: $d f$, degrees of freedom; DNHR, Danish Nationwide Health Registries; IV, inverse variance; KPNC, Kaiser Permanente Northern California; PDE5i, phosphodiesterase type 5 inhibitor; RR, risk ratio; SE, standard error.

a significantly increased risk for melanoma ( adjusted $\mathrm{RR}=1.26,95 \% \mathrm{CI}=1.07-1.50, P=0.007, I^{2}=79 \%$; Figure 4A), whereas use of tadalafil (three studies, ${ }^{17,18,20}$ adjusted $\mathrm{RR}=1.17,95 \% \mathrm{CI}=0.91-1.50, P=0.22, I^{2}=53 \%$; Figure 4A) or vardenafil (two studies, ${ }^{18,20}$ adjusted RR $=1.02$, 95\% CI $=0.90-1.16, P=0.73, I^{2}=0 \%$; Figure 4 A) was not. Finally, the stratified analysis according to the stage of melanoma revealed that PDE5i use was associated with a significantly increased risk for in situ melanoma (two studies, ${ }^{16,20}$ adjusted $\mathrm{RR}=1.31,95 \% \mathrm{CI}=1.01-1.69, P=0.04, I^{2}=69 \%$; Figure 4B), showed a trend toward increased risk for localized melanoma (three studies, ${ }^{16,20}$ adjusted $\mathrm{RR}=1.13,95 \%$ $\mathrm{CI}=0.99-1.29, P=0.06, I^{2}=23 \%$; Figure 4B), and showed a trend toward reduced risk for nonlocalized melanoma 


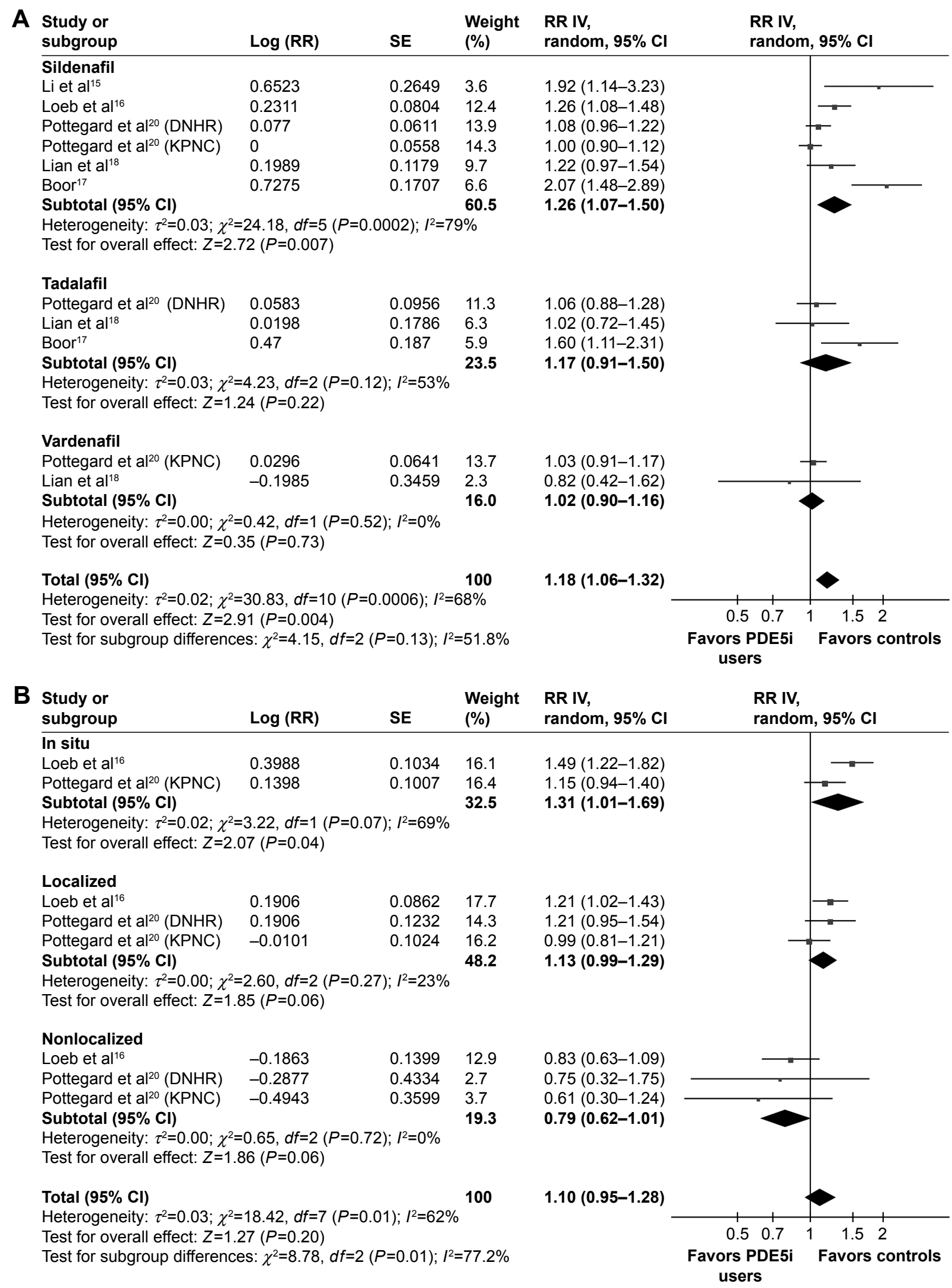

Figure 4 Forest plots for the meta-analysis of the association between PDE5i use and risk of melanoma presented as adjusted RR.

Notes: (A) Forest plots stratified by the individual PDE5i used (sildenafil, tadalafil, or vardenafil); (B) forest plots stratified by the stages of melanoma (in situ, localized, or nonlocalized melanoma). The diamond indicates the overall summary estimate for the analysis; the width of the diamond represents the $95 \% \mathrm{Cl}$.

Abbreviations: df, degrees of freedom; DNHR, Danish Nationwide Health Registries; IV, inverse variance; KPNC, Kaiser Permanente Northern California; PDE5i, phosphodiesterase type 5 inhibitor; RR, risk ratio; SE, standard error. 


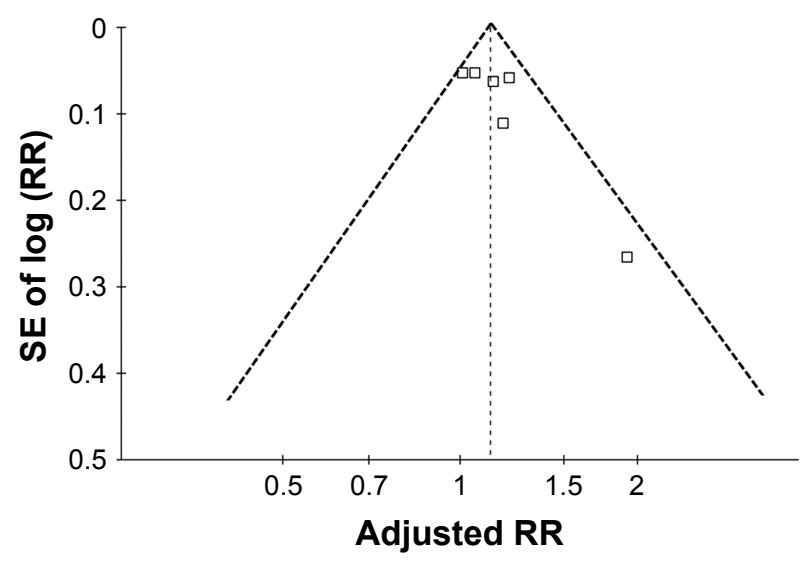

Figure 5 Funnel plot for the association between PDE5i use and risk of melanoma Notes: Each square indicates an included study. The $\mathrm{x}$-axis represents the adjusted RR of the association between PDE5i use and risk of melanoma reported in each study, and the $y$-axis represents the SE of log (RR).

Abbreviations: PDE5i, phosphodiesterase type 5 inhibitor; RR, risk ratio; SE, standard error.

(three studies, ${ }^{16,20}$ adjusted $\mathrm{RR}=0.79,95 \% \mathrm{CI}=0.62-1.01$, $P=0.06, I^{2}=0 \%$; Figure 4B).

\section{Publication bias}

The funnel plot regarding the association between PDE5i use and risk of melanoma in the overall study is shown in Figure 5. The funnel plot was symmetric on visual inspection. Based on the Egger regression test, no significant publication bias was detected for the association between PDE5i use and risk of melanoma $(P=0.623)$.

\section{Discussion}

In this meta-analysis, upon pooling the results of all available studies, we found that the use of a PDE5i was associated with an increased risk of melanoma among male participants. Moreover, subgroup analyses indicated that the association between PDE5i use and risk of melanoma remained significant in the prospective cohort studies, in participants with recent PDE5i administration, and in participants with both the lowest and highest categories of PDE5i prescriptions. These results suggest that the association between PDE5i use and risk of melanoma appears to be sequential and probably is not dose dependent. Interestingly, we also found that the use of sildenafil was associated with a significantly increased risk for melanoma, whereas use of tadalafil or vardenafil was not. Moreover, PDE5i use was associated with a significantly increased risk for in situ melanoma, but not for localized or nonlocalized melanoma. Taken together, these results demonstrate that the use of a PDE5i, particularly sildenafil, may be associated with an increased risk of melanoma in men.
Notably, although the results of our meta-analysis support the potential association between PDE5i use and risk of melanoma, at present, we could not determine whether the association between PDE5i use and melanoma is causative. The subgroup analysis in this study, which was conducted by including prospective cohort studies, only indicated that the use of PDE5i was independently associated with an increased risk of the subsequent incidence of melanoma in men, which supports a sequential relationship between PDE5i administration and melanoma incidence. This was further enhanced by analysis of adequately adjusted results. However, since the cut-offs for categorization of the number of PDE5i prescriptions varied among the included studies, we compared the association between PDE5i use and risk of melanoma in participants based on the lowest and highest prescription categories. Although these results revealed that PDE5i use was associated with an increased risk of melanoma in men with both the lowest and highest prescription categories, the differences in the results among subgroups were not statistically significant $(P$ for subgroup interaction $=0.65$ ). This finding also does not support a dose-response dependency in the association between PDE5i use and melanoma risk. To the best of our knowledge, no randomized controlled trial (RCT) has evaluated whether patients who received PDE5i have a significantly increased melanoma incidence compared to controls. Since RCTs with an adequate sample size and a sufficient follow-up duration appear to be the most reliable strategy to determine whether PDE5i is causative of melanoma incidence, these studies are warranted to confirm our results. In addition, the stratified analysis in this study regarding the use of individual PDE5i indicates that sildenafil was associated with an increased melanoma incidence, but tadalafil or vardenafil were not. However, few studies have reported the relationship between the use of tadalafil or vardenafil and risk of melanoma (only two or three studies). Therefore, the associations between other PDE5is, besides sildenafil, and risk of melanoma deserve further evaluation.

The mechanisms underlying the potential association between PDE5i use and risk of melanoma could be explained through the pharmacologic effects of PDE5i, which mimics the inhibition of cGMP-specific PDE5A induced by the activation of the RAS/RAF/ERK/MEK pathway in melanocytes. ${ }^{4}$ Direct evidence was reported in a recently published in vitro study, which discovered a cGMP-dependent growth-promoting pathway in murine and human melanoma cells and revealed that sildenafil treatment further increases intracellular cGMP concentrations, potentiating the activation 
of cell proliferation. ${ }^{14}$ However, it remains to be determined whether other PDE5is, besides sildenafil, have a similar influence on the proliferation and survival of melanocytes. Moreover, results from recent studies have suggested that the association between PDE5i use and risk of melanoma may be complicated, and the role of myeloid-derived suppressor cells (MDSCs) may be important. ${ }^{28}$ Activation of MDSCs has been associated with blocked antitumor immunity, ${ }^{28}$ while PDE5is such as sildenafil ${ }^{29}$ and tadalafil ${ }^{30,31}$ could suppress the activated MDSCs, thereby inhibiting the growth of tumor cells. The suppressive efficacy of PDE5i for MDSCs has been confirmed in melanoma-bearing animals ${ }^{30,32}$ and human patients with melanoma, ${ }^{30,33,34}$ and a recent study showed that the PDE5i tadalafil can improve the clinical outcomes for advanced melanoma patients by enhancing antitumor immunity. ${ }^{35}$ Interestingly, considering the stage of melanoma, we found that PDE5i use was associated with a significantly increased risk for in situ melanoma and also exhibited a trend toward increased risk for localized melanoma and reduced risk for nonlocalized melanoma. These findings may reflect that, for patients with advanced melanoma, PDE5i may exert an adjunctive antitumor effect by suppressing activated MDSCs. Moreover, previous studies have indicated that a BRAF somatic mutation is a major cause to the activation of the RAS/RAF/ERK/MEK pathway and the subsequent cGMP-specific PDE5A inhibition in melanocytes, which underlies the pathogenesis of melanoma related to BRAF somatic mutations. Considering that the potential stimulatory effect of PDE5i for melanoma is by mimicking the inhibitory effect of the RAS/RAF/ERK/MEK pathway for PDE5A, the carcinogenic effect of PDE5i may be insignificant in patients for whom the cGMP-specific PDE5A has already been inhibited via BRAF somatic mutation and activation of RAS/RAF/ERK/MEK signaling. Interestingly, melanoma patients with BRAF somatic mutations are more likely to have advanced melanoma with poor prognosis, as indicated by previous clinical studies ${ }^{36-38}$ and a metaanalysis. ${ }^{39}$ Therefore, the association between PDE5i use and risk of melanoma may become insignificant in patients with BRAF somatic mutations, who usually have a higher risk for advanced melanoma.

Our study has limitations that should be considered when interpreting these results. First, our meta-analysis was based on a limited number of observational studies, and we did not have access to the individual patient-based data of the original studies. Although we conducted a stratified analysis to explore the potential influence of study characteristics on the association between PDE5i use and risk of melanoma, these results should be interpreted with caution. Second, as mentioned above, the results of our study could only suggest a possible association between PDE5i use and risk of melanoma. However, future studies, preferably RCTs, are needed to determine whether this relationship is causative. Moreover, as a meta-analysis of observational studies, although we combined the most adequately adjusted results, we could exclude the possibility of residual confounding factors, such as the frequencies of BRAF somatic mutations, which may influence the results of our study. In addition, when analyzing the potential influence of PDE5i prescriptions on the association between PDE5i use and risk of melanoma, we used the amount of prescriptions, which was not as accurate as the exact dose the participants actually took. This may further confound the results. Finally, our study participants were mostly Western men. Studies should also be performed to determine the association between PDE5i use and risk of melanoma in Asians.

\section{Conclusion}

PDE5i use may be associated with a significantly increased risk of melanoma in men. However, whether the association between PDE5i and melanoma is causative requires further investigation.

\section{Author contributions}

All authors contributed to the data analysis and drafting and critically revising the paper, gave final approval of the version to be published, and agree to be accountable for all aspects of the work.

\section{Disclosure}

The authors report no conflicts of interest in this work.

\section{References}

1. Gray-Schopfer V, Wellbrock C, Marais R. Melanoma biology and new targeted therapy. Nature. 2007;445(7130):851-857.

2. Sullivan RJ, Fisher DE. Understanding the biology of melanoma and therapeutic implications. Hematol Oncol Clin North Am. 2014; 28(3):437-453.

3. Thompson JF, Scolyer RA, Kefford RF. Cutaneous melanoma in the era of molecular profiling. Lancet. 2009;374(9687):362-365.

4. Arozarena I, Sanchez-Laorden B, Packer L, et al. Oncogenic BRAF induces melanoma cell invasion by downregulating the cGMP-specific phosphodiesterase PDE5A. Cancer Cell. 2011;19(1):45-57.

5. Flaherty KT, McArthur G. BRAF, a target in melanoma: implications for solid tumor drug development. Cancer. 2010;116(21):4902-4913.

6. Packer LM, East P, Reis-Filho JS, Marais R. Identification of direct transcriptional targets of (V600E)BRAF/MEK signalling in melanoma. Pigment Cell Melanoma Res. 2009;22(6):785-798.

7. Hauschild A, Grob JJ, Demidov LV, et al. Dabrafenib in BRAF-mutated metastatic melanoma: a multicentre, open-label, phase 3 randomised controlled trial. Lancet. 2012;380(9839):358-365. 
8. Wellbrock C, Hurlstone A. BRAF as therapeutic target in melanoma. Biochem Pharmacol. 2010;80(5):561-567.

9. Palit V, Eardley I. An update on new oral PDE5 inhibitors for the treatment of erectile dysfunction. Nat Rev Urol. 2010;7(11):603-609.

10. Scaglione F, Donde S, Hassan TA, Jannini EA. Phosphodiesterase type 5 inhibitors for the treatment of erectile dysfunction: pharmacology and clinical impact of the sildenafil citrate orodispersible tablet formulation. Clin Ther. 2017;39(2):370-377.

11. Ventimiglia E, Capogrosso P, Montorsi F, Salonia A. The safety of phosphodiesterase type 5 inhibitors for erectile dysfunction. Expert Opin Drug Saf. 2016;15(2):141-152.

12. Kasumi S. In vitro effects of a phosphodiesterase inhibitor sildenafil on cellular motility of the oral malignant melanoma cells. J Oral Maxillofac Surg. 2014;72(9):e96-e97.

13. Dhayade S. CNP-derived cGMP and Sildenafil promote melanoma growth in vitro and in vivo in mice. Naunyn-Schmiedeberg's Archives of Pharmacology. 2015;388(1):S13.

14. Dhayade S, Kaesler S, Sinnberg T, et al. Sildenafil potentiates a cGMPdependent pathway to promote melanoma growth. Cell Rep. 2016; 14(11):2599-2610.

15. Li WQ, Qureshi AA, Robinson KC, Han J. Sildenafil use and increased risk of incident melanoma in US men: a prospective cohort study. JAMA Intern Med. 2014;174(6):964-970.

16. Loeb S, Folkvaljon Y, Lambe M, et al. Use of Phosphodiesterase Type 5 Inhibitors for Erectile Dysfunction and Risk of Malignant Melanoma. JAMA. 2015;313(24):2449-2455.

17. Boor P. Melanoma in men treated with PDE5A inhibitors: A report from the RADAR (research on adverse drug events and reports) project. J Am Acad Dermatol. 2016;74(5 Suppl 1):AB190.

18. Lian Y, Yin H, Pollak MN, et al. Phosphodiesterase type 5 inhibitors and the risk of melanoma skin cancer. Eur Urol. 2016;70(5):808-815.

19. Matthews A, Langan SM, Douglas IJ, Smeeth L, Bhaskaran K. Phosphodiesterase type 5 inhibitors and risk of malignant melanoma: matched cohort study using primary care data from the UK clinical practice research datalink. PLoS Med. 2016;13(6):e1002037.

20. Pottegard A, Schmidt SA, Olesen AB, et al. Use of sildenafil or other phosphodiesterase inhibitors and risk of melanoma. Br J Cancer. 2016;115(7):895-900.

21. Kearns JT, Lapin B, Wang E, et al. Associations between iCOGS single nucleotide polymorphisms and upgrading in both surgical and active surveillance cohorts of men with prostate cancer. Eur Urol. 2016; 69(2):223-228.

22. Stroup DF, Berlin JA, Morton SC, et al. Meta-analysis of observational studies in epidemiology: a proposal for reporting. Meta-analysis Of Observational Studies in Epidemiology (MOOSE) group. JAMA. 2000;283(15):2008-2012.

23. Higgins J, Green S. Cochrane Handbook for Systematic Reviews of Interventions Version 5.1.0. The Cochrane Collaboration; 2011. Available from: www.cochranehandbook.org. Accessed April 14, 2017.

24. Wells GA, Shea B, O'Connell D, et al. The Newcastle-Ottawa Scale (NOS) for assessing the quality of nonrandomised studies in meta-analyses; 2010. Available from: http://www.ohri.ca/programs/ clinical_epidemiology/oxford.asp. Accessed April 14, 2017.
25. Higgins JP, Thompson SG. Quantifying heterogeneity in a metaanalysis. Stat Med. 2002;21(11):1539-1558.

26. Patsopoulos NA, Evangelou E, Ioannidis JP. Sensitivity of betweenstudy heterogeneity in meta-analysis: proposed metrics and empirical evaluation. Int J Epidemiol. 2008;37(5):1148-1157.

27. Egger M, Davey Smith G, Schneider M, Minder C. Bias in meta-analysis detected by a simple, graphical test. BMJ. 1997;315(7109):629-634.

28. Sica A, Massarotti M. Myeloid suppressor cells in cancer and autoimmunity. J Autoimmun. Epub 2017 Jul 17.

29. Serafini P, Meckel K, Kelso M, et al. Phosphodiesterase-5 inhibition augments endogenous antitumor immunity by reducing myeloid-derived suppressor cell function. J Exp Med. 2006;203(12):2691-2702.

30. Meyer C, Sevko A, Ramacher M, et al. Chronic inflammation promotes myeloid-derived suppressor cell activation blocking antitumor immunity in transgenic mouse melanoma model. Proc Natl Acad Sci U S A. 2011; 108(41):17111-17116.

31. Noonan KA, Ghosh N, Rudraraju L, Bui M, Borrello I. Targeting immune suppression with PDE5 inhibition in end-stage multiple myeloma. Cancer Immunol Res. 2014;2(8):725-731.

32. Umansky V. Sildenafil prolongs survival and reduces immunosuppression in ret transgenic mouse melanoma model. Eur J Immunol. 2009; 39:S324.

33. Weed DT, Vella JL, Reis IM, et al. Tadalafil reduces myeloid-derived suppressor cells and regulatory $\mathrm{T}$ cells and promotes tumor immunity in patients with head and neck squamous cell carcinoma. Clin Cancer Res. 2015;21(1):39-48.

34. Califano JA, Khan Z, Noonan KA, et al. Tadalafil augments tumor specific immunity in patients with head and neck squamous cell carcinoma. Clin Cancer Res. 2015;21(1):30-38.

35. Hassel JC, Jiang H, Bender C, et al. Tadalafil has biologic activity in human melanoma. Results of a pilot trial with Tadalafil in patients with metastatic Melanoma (TaMe). Oncoimmunology. 2017;6(9): e1326440.

36. Barbour AP, Tang YH, Armour N, et al. BRAF mutation status is an independent prognostic factor for resected stage IIIB and IIIC melanoma: implications for melanoma staging and adjuvant therapy. Eur $J$ Cancer. 2014;50(15):2668-2676.

37. Santiago-Walker A, Gagnon R, Mazumdar J, et al. Correlation of BRAF mutation status in circulating-free DNA and tumor and association with clinical outcome across four BRAFi and MEKi clinical trials. Clin Cancer Res. 2016;22(3):567-574

38. Siroy AE, Aung PP, Torres-Cabala CA, et al. Clinical significance of BRAF V600E mutational status in capsular nevi of sentinel lymph nodes in patients with primary cutaneous melanoma. Hum Pathol. 2017; 59:48-54.

39. Kim SY, Kim SN, Hahn HJ, Lee YW, Choe YB, Ahn KJ. Metaanalysis of BRAF mutations and clinicopathologic characteristics in primary melanoma. J Am Acad Dermatol. 2015;72(6):1036-1046.e2.
OncoTargets and Therapy

\section{Publish your work in this journal}

OncoTargets and Therapy is an international, peer-reviewed, open access journal focusing on the pathological basis of all cancers, potential targets for therapy and treatment protocols employed to improve the management of cancer patients. The journal also focuses on the impact of management programs and new therapeutic agents and protocols on
Dovepress

patient perspectives such as quality of life, adherence and satisfaction The manuscript management system is completely online and includes a very quick and fair peer-review system, which is all easy to use. Visit http://www.dovepress.com/testimonials.php to read real quotes from published authors. 